\title{
Dl-3n-butylphthalide improves spatial learning and memory in rats with vascular dementia by reducing autophagy via regulation of the $m$ TOR signaling pathway
}

\author{
AYONG TIAN ${ }^{1}$, XIAOCHUAN MA ${ }^{2}$, HUI LI $^{3}$ and RONGWEI ZHANG ${ }^{3}$ \\ ${ }^{1}$ Department of Anesthesiology, The First Affiliated Hospital of China Medical University; \\ ${ }^{2}$ Department of Gerontology and Geriatrics, The Third Hospital of Shenyang; ${ }^{3}$ Department of Gerontology and \\ Geriatrics, The First Affiliated Hospital of China Medical University, Shenyang, Liaoning 110001, P.R. China
}

Received February 8, 2019; Accepted November 8, 2019

DOI: $10.3892 /$ etm.2019.8402

\begin{abstract}
Dl-3n-butylphthalide (NBP) has been reported to be a beneficial and promising drug for the treatment and prevention of vascular dementia (VD). NBP has been demonstrated to improve learning and memory in rats with vascular cognitive impairment by activating the silent information regulator $1 /$ brain-derived neurotrophic factor pathway. However, NBP is a multi-target drug. Therefore, the present study aimed to determine whether the protective effects of NBP on learning deficits in a rat model of VD were due to the inhibition of autophagy via the phosphorylated mammalian target of rapamycin (p-mTOR) pathway. NBP treatment attenuated memory damage in rats with VD, as demonstrated by T-maze and Morris water maze tests. NBP administration also significantly reduced the levels of the characteristic autophagic proteins Beclin 1 and LC3II and upregulated phosphorylation levels of mTOR at Ser-2448 compared with the VD group. However, treatment of rats with VD with NBP plus the mTOR inhibitor rapamycin failed to significantly suppress Beclin 1 and LC3II expression. These results suggested that the beneficial effects of NBP on learning deficits in a rat model of VD were due to the suppression of ischemia-induced autophagy via the p-mTOR signaling pathway.
\end{abstract}

\section{Introduction}

Vascular dementia (VD) is one of the most common types of dementia after Alzheimer's disease, accounting for around $15 \%$ of cases (1) and characterized by a progressive decline in memory and learning (2). Accumulating evidence suggests

Correspondence to: Professor Rongwei Zhang, Department of Gerontology and Geriatrics, The First Affiliated Hospital of China Medical University, 155 Nanjing North Street, Heping, Shenyang, Liaoning 110001, P.R. China

E-mail: rongweizhang@126.com

Key words: Dl-3n-butylphthalide, dementia, autophagy, rapamycin that vascular risk factors may contribute to the onset of VD (3). However, there are currently no licensed treatments for VD and the mechanisms underlying its pathogenesis remain unclear.

Autophagy is a cell self-degradation process that is important for maintaining the stability of the internal environment of the body (4) by clearing damaged cellular components, such as mitochondria (5). However, overactivation of autophagy triggers cell death as a result of excessive self-digestion through the degradation of essential proteins and organelles (6). Previous studies have reported that activation of autophagy as a result of transient ischemia promotes neuronal damage in brain tissues $(7,8)$, suggesting that autophagy is a common pathway leading to cell death in the central nervous system.

3-n-butylphthalide (NBP), initially extracted from the seeds of Chinese celery (Apium gravelens), has been approved for the treatment of ischemic cerebrovascular disease (9). Based on its multi-target therapeutic properties, NBP has demonstrated an important role in a number of nervous system diseases, including amyotrophic lateral sclerosis (10), Parkinson's disease (11) and VD (12), as well as models of Alzheimer's disease (13). Studies have also demonstrated that multiple mechanisms are involved in the neuroprotective effects of NBP, including anti-inflammatory effects, suppression of oxidative stress, inhibition of platelet aggregation and anti-apoptosis (14-17). However, little is known about the protective role of NBP against chronic ischemia-induced excessive autophagy in VD. The present study aimed to investigate the effect of NBP on autophagy in the hippocampus of a rat model of VD and to determine the signaling pathways involved in the observed effects.

\section{Materials and methods}

Animals and groups. A total of 60 male Sprague-Dawley rats (age, 2 months; weight, 250-280 g) were purchased from the Experimental Animal Center of China Medical University (Shenyang, China). All rats were housed in a specific pathogen-free animal experiment room at $24 \pm 2{ }^{\circ} \mathrm{C}$ with $60 \%$ humidity under a 12-h light/dark cycle and were allowed free access to water and food. The experiments 
were approved by the China Medical University Animal Care and Use Committee and adhered to the Chinese Academy of Science guidelines for the care and use of laboratory animals. All rats were randomly divided into five groups (n=12 rats/group): i) Sham (S) group; ii) VD group; iii) NBP (N) group; iv) rapamycin (R) group; and v) NBP and rapamycin $(\mathrm{N}+\mathrm{R})$ group. One day prior to the surgery, rats in the $\mathrm{R}$ and $\mathrm{N}+\mathrm{R}$ groups underwent lateral ventricle catheterization and $50 \mu \mathrm{rapamycin}(1 \mathrm{mmol} / \mathrm{ml})$ was injected slowly into the lateral ventricle $(2 \mu \mathrm{l} / \mathrm{min})$, leaving the needle in for $5 \mathrm{~min}$. With the exception of the $\mathrm{S}$ group, all rats underwent vessel ligation. VD was induced by two-vessel occlusion as previously described (18). Sham rats were subjected to the same procedure without ligation of the arteries. Rats in the $\mathrm{S}$ and VD groups received vegetable oil, and the other groups received $60 \mathrm{mg} / \mathrm{kg}$ NBP per day. All rats were weighed daily. Four weeks after the surgery, there were 12 rats in the $S$ group, 10 in the VD group, 11 in the $\mathrm{N}$ group, 10 in the $\mathrm{R}$ group and 11 in the $\mathrm{N}+\mathrm{R}$ group. A total of six rats were excluded from the study due to epilepsy in two rats and death of unexplained causes in four rats. NBP soft capsules were purchased from Shijiazhuang Pharmaceutical Co. Ltd. The study timeline is presented in Fig. 1.

Behavioral tests T-maze. T-maze tests can be used to assess an animal's spatial working memory (19). T-maze tests were performed after 4 weeks of NBP treatment (S, $n=12$; VD, $n=10$; $\mathrm{N}, \mathrm{n}=11 ; \mathrm{R}, \mathrm{n}=11 ; \mathrm{N}+\mathrm{R}, \mathrm{n}=11)$. Each T-maze trial consisted of a sample run and a choice run. On the sample run, rats were forced to enter either the left or the right arm of the maze to get sugar, while the other arm was blocked by a sliding door. On the choice run, the blocked door was removed and the rats were allowed to choose either arm freely. There was an interval of $10 \mathrm{sec}$ between the sample and choice runs. If the rats entered the previously unvisited arm, they were rewarded. The delay was then prolonged to 90 and $180 \mathrm{sec}$. Each daily session consisted of five trials, and rats performed one trial at a time with an interval of $10 \mathrm{~min}$. The number of corrections made when the rats entered the unvisited arm of the T-maze was measured.

Morris water maze (MWM). The MWM test was performed in a circular pool (diameter, $180 \mathrm{~cm}$; height, $60 \mathrm{~cm}$; depth, $35 \mathrm{~cm}$ ) filled with opaque water by stirring in milk (temperature, $\left.26 \pm 1^{\circ} \mathrm{C}\right)$. The pool was divided into four quadrants, and a white platform was located in the center of one quadrant. The rat was gently placed in the water, facing the side-wall of the maze not housing the platform. The time to find the hidden platform (escape latency) and path length (distance traveled) were recorded for each training trial. The rat was given a maximum of $90 \mathrm{sec}$ to find the hidden platform, any rat who failed the mission within $90 \mathrm{sec}$ would be guided to the hidden platform and allowed to stay on the platform for $15 \mathrm{sec}$, before the training was terminated. The rats started each trial from a different quadrant and were trained twice a day for 5 consecutive days with an interval of $3 \mathrm{~h}$. The escape latency and swimming distances were recorded using a video camera, and the digital images were analyzed using water maze software (HVS Image 2020; HVS Image Software Ltd.) Additional probe trials were conducted with the hidden platform removed on the 6th day of the test. Swimming speed, times of crossing the platform, time spent and swimming distance in the target quadrant were also recorded.

Nissl staining. Rats were euthanized with an intraperitoneal injection of sodium pentobarbital $(200 \mathrm{mg} / \mathrm{kg})$; when continuous spontaneous breathing arrest for 2-3 min and muscle relaxation occurred, rats were transcardially perfused with normal saline $(0.9 \%)$ followed by $4 \%$ paraformaldehyde in $0.1 \mathrm{M}$ sodium phosphate buffer ( $\mathrm{pH} 7.3)$. The brain was then dissected, postfixed with $4 \%$ paraformaldehyde at $4^{\circ} \mathrm{C}$ for three days, embedded in paraffin, cut into $5-\mu \mathrm{m}$ sections and stained with $1 \%$ Toluidine Blue at $60^{\circ} \mathrm{C}$ for $40 \mathrm{~min}$. Nissl-positive cells in the hippocampus were examined to assess neuronal survival. The population of normal neurons in the CA1 subfield was counted using a light microscope at x400 magnification (model, BX53; Olympus Corporation) by two independent investigators who were blinded to the experimental conditions. The mean number of neurons was obtained by counting 3 sections per brain and 5 representative fields were randomly selected to count per section.

Transmission electron microscopy. Brain tissues were prepared for electron microscopy by fixing in $2.5 \%$ glutaraldehyde in $\mathrm{PBS}\left(\mathrm{pH} \mathrm{7.4)}\right.$ ) for $1 \mathrm{~h}$ at $4^{\circ} \mathrm{C}$ and in $1 \% \mathrm{OsO}_{4}$ in $0.1 \mathrm{~mol} / 1$ cacodylate buffer ( $\mathrm{pH}$ 7.4) for $2 \mathrm{~h}$ at $4^{\circ} \mathrm{C}$ The tissues were subsequently stained with $1 \%$ aqueous uranyl acetate at $4^{\circ} \mathrm{C}$ overnight and embedded in Durcopan (Sigma-Aldrich, Merck $\mathrm{KGaA}$ ). The sections (50-70 nm) of the hippocampus were cut using a Leica ultracut microtome (Leica Microsystems, Inc.) and collected on formvar-coated copper grids. The sections were stained with $1 \%$ aqueous uranyl acetate and lead citrate and then imaged on a $\mathrm{H}-600 / 7650$ transmission electron microscope (Hitachi High-Technologies Corporation).

Western blotting. Rats were euthanized with an intraperitoneal injection of sodium pentobarbital $(200 \mathrm{mg} / \mathrm{kg})$ and sacrificed by transcardiac perfusion with normal saline $(0.9 \%)$ followed by $4 \%$ paraformaldehyde in $0.1 \mathrm{M}$ sodium phosphate buffer ( $\mathrm{pH}$ 7.3). The rat ( $\mathrm{n}=5 /$ group) hippocampus was homogenized in RIPA lysis buffer (cat. no. P0013B; Beyotime Institute of Biotechnology) containing PMSF, and centrifuged at $12,000 \mathrm{x} \mathrm{g}$ for $10 \mathrm{~min}$ at $4^{\circ} \mathrm{C}$. Protein concentrations were determined using a bicinchoninic acid protein assay kit (Beyotime Institute of Biotechnology). A total of $30 \mu \mathrm{g}$ of protein per lane was separated by $12 \%$ SDS-PAGE and transferred onto PVDF membranes. The membranes were blocked with $3 \%$ bovine serum albumin (Sigma-Aldrich; Merck KGaA) in Tris-buffered saline at room temperature for $30 \mathrm{~min}$ and incubated overnight at $4^{\circ} \mathrm{C}$ with primary antibodies against mammalian target of rapamycin (mTOR; 1:400; cat. no. 2972; Cell Signaling Technology, Inc.), phosphorylated (p)-mTOR (Ser2448; 1:250; cat. no. 2971; Cell Signaling Technology, Inc.), LC3B (1:1,000; cat. no. 2775; Cell Signaling Technology, Inc.), Beclin 1 (1:1,000; cat. no. sc-48341; Santa Cruz Biotechnology, Inc.) or $\beta$-actin (1:1,000, cat. no. 4967; Cell Signaling Technology, Inc.). Following incubation with horseradish-peroxidase conjugated anti-rabbit IgG (1:2,000; Cell Signaling Technology, Inc.) for $1 \mathrm{~h}$ at room temperature. Protein signals were detected using an enhanced chemiluminescence system (EMD Millipore) and 


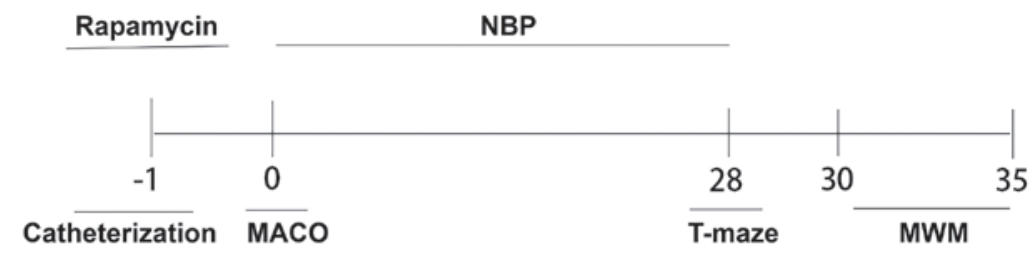

Figure 1. Experimental timeline.
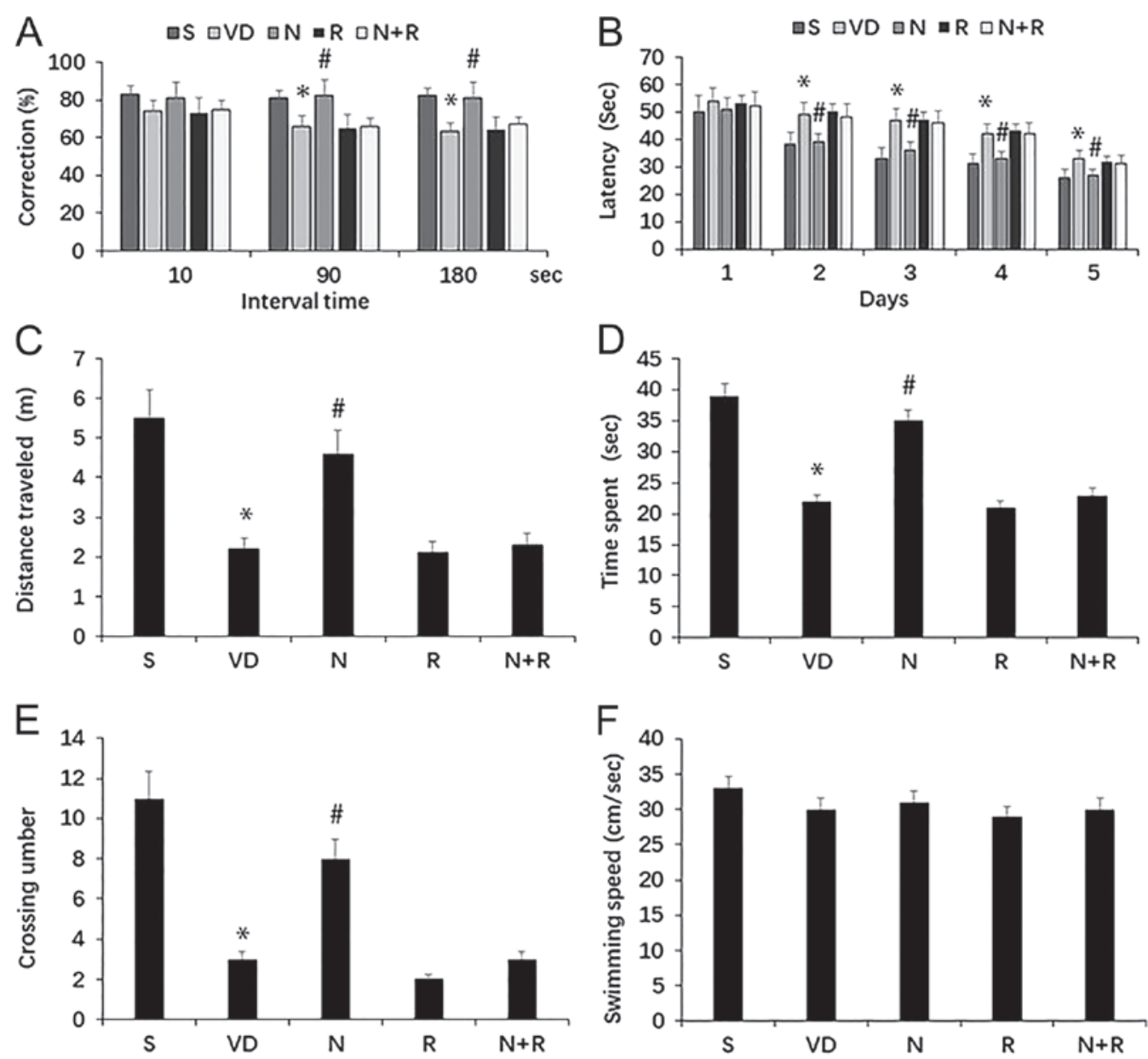

Figure 2. Effects of NBP on ischemia-induced cognitive decline in the T-maze and MWM tests. (A) Correction alternation of various intervals with a 10,90 and $180 \mathrm{sec}$ delay in the T-maze. (B) Escape latency in the MWM test. (C) Distance travelled in the MWM test. (D) Time spent in the target quadrant in MWM test. (E) Platform crossing counts in the MWM test. (F) Swimming speed in the MWM test. Data are expressed as the means \pm SEM. ${ }^{\circ}<0.05$ vs. $\mathrm{S}$ group; ${ }^{*} \mathrm{P}<0.05$ vs. VD group. S, sham; VD, vascular dementia; N, NBP; N+R, NBP + rapamycin; NBP, Dl-3n-butylphthalide; MWM, Morris water maze.

quantified using Quantity-One software version 4.6.3 (Bio-Rad Laboratories, Inc.). $\beta$-actin was used as a protein-loading control.

Statistical analysis. Data are presented as the mean \pm standard deviation and were analyzed using SPSS 16.0 statistical software (SPSS, Inc.). Statistical significance was determined by one-way ANOVA followed by the least significant difference post hoc test. $\mathrm{P}<0.05$ was considered to indicate a statistically significant difference.

\section{Results}

NBP ameliorates autophagy-induced learning and memory injury in rats with $V D$. The cognitive function of rats with VD was first assessed using the T-maze test. Rats in the VD group exhibited impaired spontaneous alternation behavior compared with those in the $\mathrm{S}$ group $(\mathrm{P}<0.05)$; however, NBP attenuated this impairment in rats with $\mathrm{VD}(\mathrm{P}<0.05$; Fig. $2 \mathrm{~A})$. NBP also improved the performance of VD rats when the interval between the sample and choice runs was delayed by 90 and $180 \mathrm{sec}$ (both $\mathrm{P}<0.05$ ). In addition, compared with the $\mathrm{R}$ group, rats in the $\mathrm{N}+\mathrm{R}$ group exhibited no improvement in performance in terms of spontaneous alternation behavior, including when the interval was delayed by 90 and $180 \mathrm{sec}$ ( $>00.05$; Fig. 2A).

Cognitive function was determined using the MWM test. During training, significant differences in the performance of the five rat groups were observed from day 2; rats in the VD group exhibited a higher escape latency compared with the $\mathrm{S}$ group $(\mathrm{P}<0.05)$, and the escape latency was shortened 
A $s$

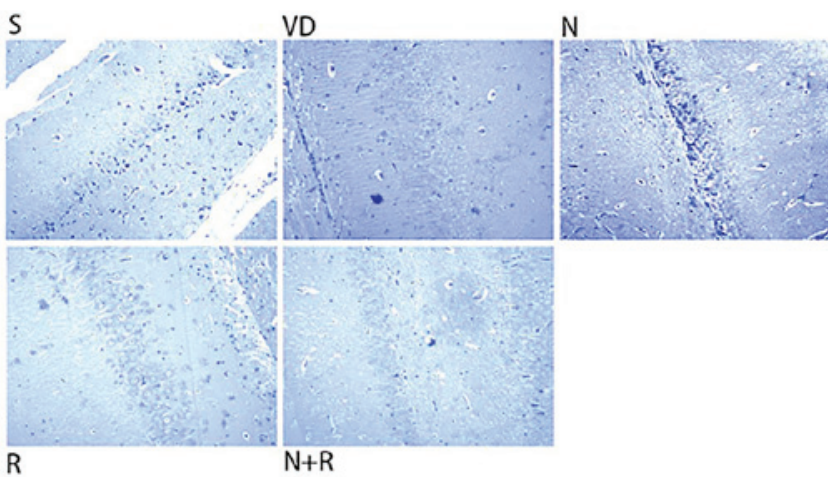

B

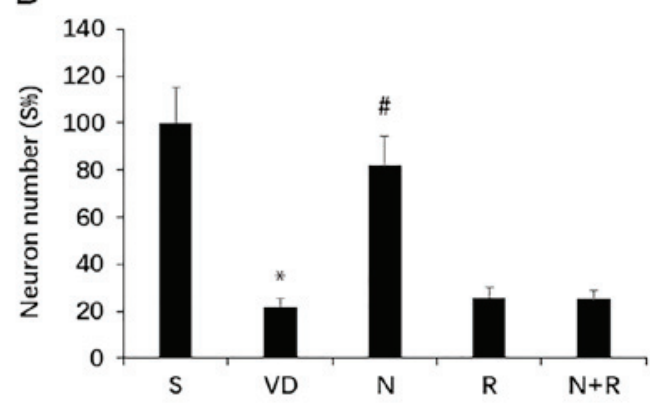

Figure 3. Effects of NBP on neuronal survival in the CA1 region of the hippocampus. (A) Hippocampal neuron survival was detected using Nissl staining (magnification, $\mathrm{x} 400$ ), and (B) the relative number of neurons with Nissl bodies was obtained. Data are expressed as the means $\pm \mathrm{SEM}$. ${ }^{*}<0.05$ vs. $\mathrm{S}$ group; ${ }^{\#} \mathrm{P}<0.05$ vs. VD group. S, sham; VD, vascular dementia; N, NBP; N+R, NBP + rapamycin; NBP, Dl-3n-butylphthalide.
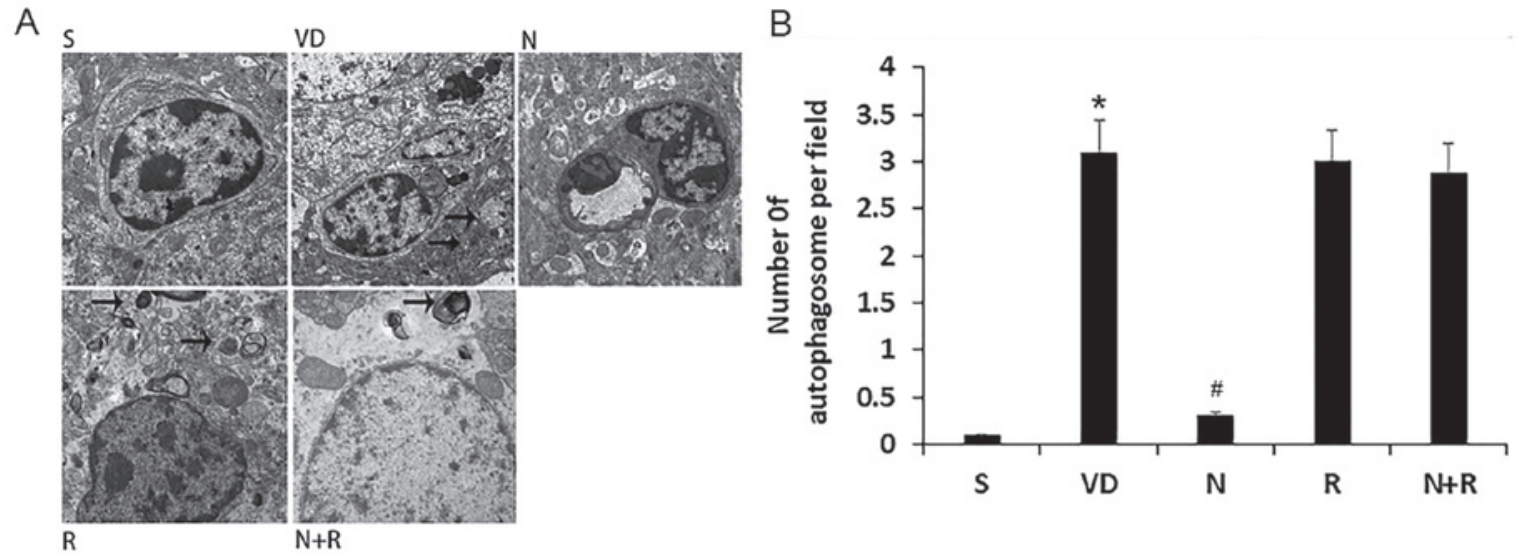

Figure 4. Effects of NBP on autophagosome formation in the CA1 area of the hippocampus. (A) Electron microscopic examination (magnification, x10,000) of autophagosomes in the CA1 area. Normal ultrastructure was observed in the S group, and scattered autophagosomes were observed in the VD, R and $\mathrm{N}+\mathrm{R}$ groups. A low number of autophagosomes was observed in the $\mathrm{N}$ group. Arrow marked the autophagosomes. (B) Quantitative analysis of the number of autophagosomes of per field. Data are expressed as means \pm SEM. ${ }^{*} \mathrm{P}<0.05$ vs. S group, ${ }^{\#} \mathrm{P}<0.05$ vs. VD group. S, sham; VD, vascular dementia; N, NBP; N+R, $\mathrm{NBP}+$ rapamycin; NBP, D1-3n-butylphthalide.

by treatment with NBP $(\mathrm{P}<0.05 ;$ Fig. $2 \mathrm{~B})$. In the probe test, the time spent swimming and the distance in the target quadrant, as well as the number of platform crossings were significantly lower in the VD group compared with the $\mathrm{S}$ group (all $\mathrm{P}<0.05)$. NBP increased the time spent swimming, the distance in the target quadrant and the number of platforms crossed compared with the VD group (all $\mathrm{P}<0.05$ ). However, the $\mathrm{N}+\mathrm{R}$ group exhibited no change in time spent swimming, distance in the target quadrant or the number of platform crossings compared with the R group (all P>0.05; Fig. 2C-E). No difference was observed in swimming speed among the groups $(\mathrm{P}>0.05$; Fig. 2F). These results indicated that NBP ameliorated the impairment of learning and memory induced by ischemia, but did not improve rapamycin-aggravated learning and memory deficits in rats with VD (Fig. 2).

NBP promotes neuronal survival in rats with VD. No histopathological abnormalities were observed in the hippocampus in rats in the $\mathrm{S}$ group, but VD rats exhibited marked neuronal loss and degeneration of neuronal structure in the CA1 region(Fig. 3). By contrast,NBP attenuated this loss and reversed the structural injury, reflected by the density and morphology of neurons with Nissl bodies $(\mathrm{P}<0.05)$. Histopathological abnormalities detected by Nissl staining were similar in the $\mathrm{N}+\mathrm{R}$ and $\mathrm{R}$ groups $(\mathrm{P}>0.05)$. These results suggested that NBP may inhibit hippocampal neuron death in rats with VD, but could not reverse the injury in rapamycin-treated rats.

NBP decreases autophagosome formation in the CAl area of the hippocampus in rats with VD. No autophagosomes were visible in the hippocampal CA1 region of rats in the $S$ group under transmission electron microscopy (Fig. 4). By contrast, round autophagosomes were located next to the nuclei in the VD group, and there were abundant primary lysosomes, which indicated autophagy. Fewer autophagosomes were observed in the NBP group. Similarly to the VD group, autophagosomes and lysosomes were abundant in the $\mathrm{R}$ and $\mathrm{N}+\mathrm{R}$ groups. These results suggested that NBP may inhibit autophagosome formation in rats with VD (Fig. 4).

NBP inhibits the expression of characteristic autophagy proteins and increases $m T O R$ phosphorylation in rats with VD. The changes in Beclin 1, LC3II and p-mTOR protein expression levels were determined by western blot analysis (Fig. 5). 

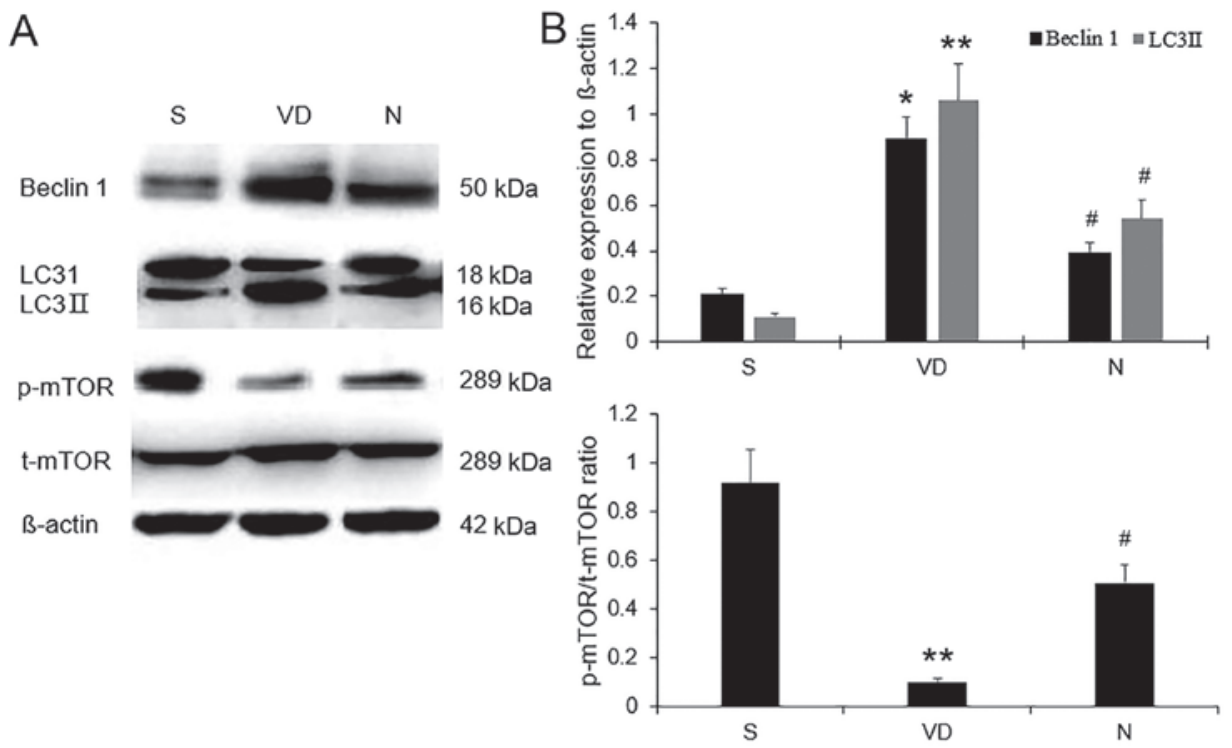

Figure 5. Effects of NBP on autophagy-related protein expression in the CA1 area of the hippocampus. (A) The protein expression levels of Beclin 1, LC3II and p-mTOR in the CA1 area were examined by western blot analysis. (B) Relative densitometric analysis of Beclin 1, LC3II and p-mTOR protein bands. Data are expressed as the means \pm SEM. ${ }^{*} \mathrm{P}<0.05$ and ${ }^{* *} \mathrm{P}<0.01$ vs. $\mathrm{S}$; ${ }^{*} \mathrm{P}<0.05$ vs. VD. S, sham group; VD, vascular dementia group; N, NBP group; NBP, Dl-3n-butylphthalide; p, phosphorylated; t, total; LC3, microtubule-associated protein 1A/1B-light chain 3.

A
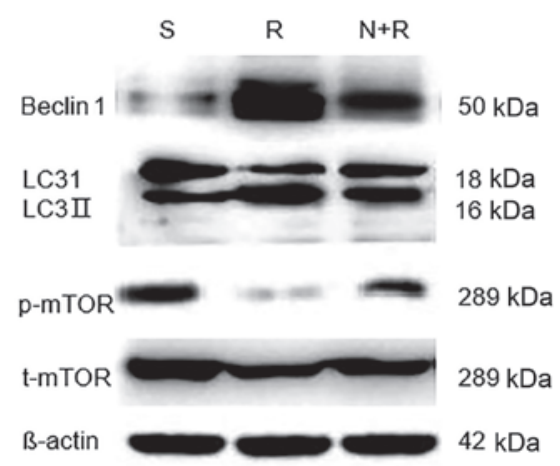

$\mathrm{B}$
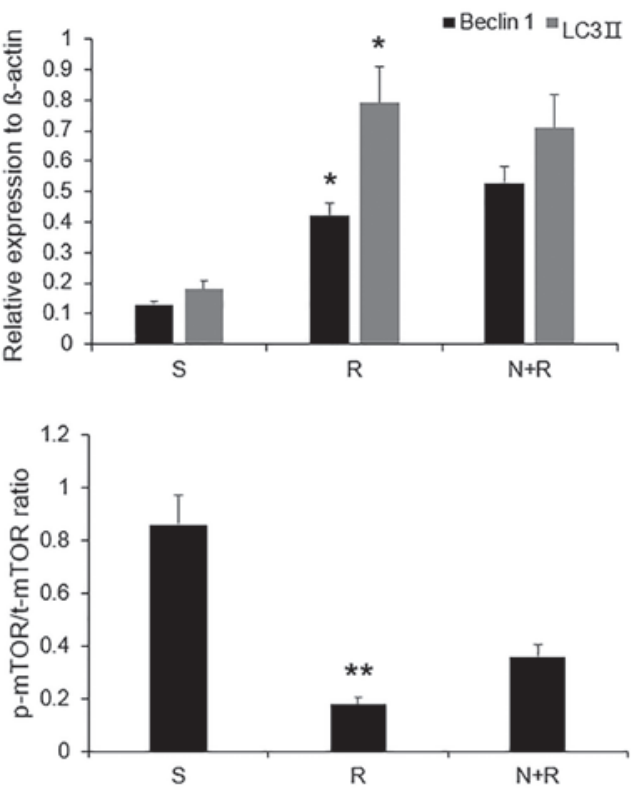

Figure 6. Effects of NBP on the mTOR signaling pathway in the CA1 area of the hippocampus. (A) The protein expression levels of Beclin 1, LC3II and p-mTOR in CA1 following the inhibition of the mTOR signaling pathway were measured by western blot analysis. (B) Relative densitometric analysis of Beclin 1, LC3II and p-mTOR protein bands. Data are expressed as the means \pm SEM. ${ }^{*} \mathrm{P}<0.05$ and ${ }^{* *} \mathrm{P}<0.01$ vs. S. S, sham group; R, rapamycin group; N+R, $\mathrm{NBP}+$ rapamycin group; NBP, Dl-3n-butylphthalide; p, phosphorylated; t, total; LC3, microtubule-associated protein 1A/1B-light chain 3.

Low levels of Beclin 1 and LC3II were present in the hippocampal CA1 region in rats in the $\mathrm{S}$ group. However, expression levels of Beclin 1 and LC3II were significantly increased in the VD group $(\mathrm{P}<0.05$ and $\mathrm{P}<0.01$, respectively) but reduced in the NBP group (both $\mathrm{P}<0.05$ ). By contrast, the levels of p-mTOR Ser-2448 in the S group were higher compared with those in the VD group $(\mathrm{P}<0.01)$, and this reduced phosphorylation of mTOR was partially reversed by NBP administration in rats with $\mathrm{VD}(\mathrm{P}<0.05)$. These results demonstrated that NBP partially inhibited excessive ischemia-induced autophagy and promoted mTOR phosphorylation in rats with VD.
NBP inhibits autophagy via an mTOR-dependent pathway in rats with $V D$. The role of $\mathrm{mTOR}$ in ischemia-induced autophagy was assessed by measuring mTOR phosphorylation levels in the five groups. The administration of the mTOR inhibitor rapamycin reduced mTOR phosphorylation $(\mathrm{P}<0.01)$ and simultaneously increased the expression levels of Beclin 1 and LC3II in VD rats compared with the $\mathrm{S}$ group (both $\mathrm{P}<0.05)$, whereas NBP + rapamycin failed to reverse these effects compared with rapamycin alone ( $\mathrm{P}>0.05$; Fig. 6). These results indicated that NBP may suppress autophagy through an mTOR-dependent pathway in rats with VD. 


\section{Discussion}

VD is a common cause of dementia, primarily induced by cerebrovascular disease or risk factors including age, hypertension, diabetes, and smoking (20). An increasing number of studies recently focused on the pathological mechanisms and possible therapies for VD; however, VD remains difficult to treat, and further studies are therefore urgently needed.

Autophagy, which is an adaptive reaction of cells to stress produced by alterations in the internal and external environments, is a dynamic catabolic process involved in multiple cellular processes (21). However, excessive autophagy may trigger cell death and contribute to ischemia-induced neuronal damage $(22,23)$. Inhibition of autophagy has thus demonstrated beneficial effects in modulating neurological deficits following cerebral ischemia $(24,25)$.

The kinase mTOR is a common regulator of autophagy induction; mTOR activation suppresses, whereas inhibiting mTOR promotes autophagy (26). Rapamycin is a direct inhibitor of mTOR and is the most commonly used and specific inducer of autophagy (27). The mTOR pathway activation has been demonstrated to protect hippocampal neurons against hypoxia-induced injury and promote neuronal recovery (28).

In the present study, a rat model of VD was established (29), and learning and memory in the rats were evaluated by conducting MWM and T-maze tests. The results demonstrated that NBP significantly improved the cognitive performance of rats with VD, but could not attenuate cognitive decline in rats with VD following treatment with the autophagy agonist rapamycin. These results, along with the changes in the relative numbers of Nissl-positive cells in the CA1 region of the hippocampus in the NBP-treated or the NBP combined with rapamycin treated group, indicated that NBP could ameliorate cognitive impairment and neuronal loss in rats with VD, and that these effects of NBP could be prevented by the autophagy agonist rapamycin.

Numerous studies have reported that autophagy is activated in neurons subjected to ischemia or hypoxia, and excessive autophagy may lead to autophagic neuronal cell death (30-33). The results of the transmission electron microscopy analysis in the present study revealed that brain ischemia induced autophagosome formation in VD rats, which was inhibited by NBP. This NBP-induced suppression of autophagy was confirmed by western blot analysis, which revealed that the expression levels of the autophagy markers LC3II and Beclin 1 were downregulated in NBP-treated rats with VD, indicating that inhibition of autophagy by NBP may prevent the learning and memory impairments observed in rats with VD.

The upstream signaling pathway of ischemia-mediated autophagy activation and the role of this pathway in the inhibitory effect of NBP on autophagy were further elucidated by detecting mTOR phosphorylation levels in rats with VD. mTOR a negative regulator of autophagy, and mTOR phosphorylation levels in rats with VD were notably enhanced by NBP treatment, accompanied by a significant downregulation of autophagy protein expression. In addition, mTOR phosphorylation decreased, whereas the expression levels of autophagy-related proteins increased in rats with
VD following injection of the mTOR inhibitor rapamycin; however, NBP co-treatment with rapamycin did not enhance mTOR phosphorylation or inhibit autophagy-related protein expression in rats with VD. These results indicated that excessive autophagy may occur in rats with VD and may trigger neuronal damage, which was consistent with previous studies $(21,22)$. This process may be reversed by NBP through upregulating mTOR phosphorylation; blocking mTOR signaling using the specific inhibitor rapamycin prevented the reversal of autophagy by NBP in rats with VD, suggesting that the neuroprotective effect of NBP may be mTOR-dependent.

In conclusion, the results of the present study demonstrated that NBP attenuated cognitive decline and neuronal loss in the CA1 region of the hippocampus in rats exposed to cerebral ischemia. In addition, the beneficial effects of NBP were associated with the activation of the mTOR signaling pathway and suppression of autophagy-related protein expression. However, this study had certain limitations. In vivo, the influence of other factors could not be completely ruled out; it is not clear whether the accumulated autophagic vacuoles in rats with VD were the result of autophagy induction or autophagic flux impairment, and whether NBP exerted its effects on autophagy induction or autophagic flux. In our future research, rat hippocampal neuron cells will be cultured to measure the influence of NBP on autophagy induction and the state of autophagic flux in vitro, which may elucidate the detailed mechanisms by which NBP exerts a neuroprotective effect in rats with VD.

\section{Acknowledgements}

Not applicable.

\section{Funding}

This study was supported by National Key R\&D Program of China (grant no. 2018YFC1311600), Liaoning Province Nature Science Foundation of China (grant no. 2015020471) and Science and Technology Program of Shenyang (no. 17-230-930)

\section{Availability of data and materials}

The datasets used and/or analyzed during the current study are available from the corresponding author on reasonable request.

\section{Authors' contributions}

AT wrote the manuscript and performed statistical analysis. RZ and AT designed the study. XM established the rat models. $\mathrm{XM}$ and HL collected and analyzed all experimental data. All authors have read and approved the final manuscript.

\section{Ethics approval and consent to participate}

The experiments performed in the present study were approved by The Animal Research Committee of the First Affiliated Hospital of China Medical University. 


\section{Patient consent for publication}

Not applicable.

\section{Competing interests}

The authors declare that they have no competing interests.

\section{References}

1. O'Brien JT and Thomas A: Vascular dementia. Lancet 386: 1698-1706, 2015.

2. Smith EE: Clinical presentations and epidemiology of vascular dementia. Clin Sci (Lond) 131: 1059-1068, 2017.

3. Jayant S and Sharma B: Selective modulator of cannabinoid receptor type 2 reduces memory impairment and infarct size during cerebral hypoperfusion and vascular dementia. Curr Neurovasc Res 13: 289-302, 2016.

4. Voigt $\mathrm{O}$ and Pöggeler S: Self-eating to grow and kill: Autophagy in filamentous ascomycetes. Appl Microbiol Biotechnol 97: 9277-9290, 2013.

5. Bharadwaj PR, Verdile G, Barr RK, Gupta V, Steele JW, Lachenmayer ML, Yue Z, Ehrlich ME, Petsko G, Ju S, et al: Latrepirdine (dimebon) enhances autophagy and reduces intracellular GFP-A $\beta 42$ levels in yeast. J Alzheimers Dis 32: 949-967, 2012.

6. Ouyang L, Shi Z, Zhao S, Wang FT, Zhou TT, Liu B and Bao JK: Programmed cell death pathways in cancer: A review of apoptosis, autophagy and programmed necrosis. Cell Prolif 45: 487-498, 2012.

7. Lu J, Qian HY, Liu LJ, Zhou BC, Xiao Y, Mao JN, An GY, Rui MZ, Wang T and Zhu CL: Mild hypothermia alleviates excessive autophagy and mitophagy in a rat model of asphyxial cardiac arrest. Neurol Sci 35: 1691-1699, 2014.

8. Fujita S, Sakurai M, Baba H, Abe K and Tominaga R: Autophagy-mediated stress response in motor neurons after hypothermic spinal cord ischemia in rabbits. J Vasc Surg 62 : $1312-1319,2015$

9. Abdoulaye IA and Yi JG: A review of recent advances in neuroprotective potential of 3-N-butylphthalide and its derivatives. Biomed Res Int 2016: 5012341, 2016.

10. Feng X, Peng Y, Liu M and Cui L: DL-3-n-butylphthalide extends survival by attenuating glial activation in a mouse model of amyotrophic lateral sclerosis. Neuropharmacology 62: 1004-1010, 2012

11. Xiong N, Huang J, Chen C, Zhao Y, Zhang Z, Jia M, Zhang Z Hou L, Yang H, Cao X, et al: Dl-3-n-butylphthalide, a natura antioxidant, protects dopamine neurons in rotenone models for Parkinson's disease. Neurobiol Aging : 1777-1791, 2012.

12. Zhu J, Wang Y, Li J, Deng J and Zhou H: Intracranial artery stenosis and progression from mild cognitive impairment to Alzheimer disease. Neurology 82: 842-849, 2014.

13. Peng Y, Sun J, Hon S, Nylander AN, Xia W, Feng Y, Wang X and Lemere CA: L-3-n-butylphthalide improves cognitive impairment and reduces amyloid-beta in a transgenic model of Alzheimer's disease. J Neurosci 30: 8180-8189, 2010.

14. He Z, Zhou Y, Lin L, Wang Q, Khor S, Mao Y, Li J, Zhen Z, Chen J, Gao Z, et al: Dl-3-n-butylphthalide attenuates acute inflammatory activation in rats with spinal cord injury by inhibiting microglial TLR4/NF- $\kappa \mathrm{B}$ signalling. J Cell Mol Med 21: 3010-3022, 2017

15. Sheng X, Hua K, Yang C, Wang X, Ji H, Xu J, Huang Z and Zhang Y: Novel hybrids of 3-n-butylphthalide and edaravone: Design, synthesis and evaluations as potential anti-ischemic stroke agents. Bioorg Med Chem Lett 25: 3535-3540, 2015.
16. Wang F, Ma J, Han F, Guo X, Meng L, Sun Y, Jin C, Duan H, Li $\mathrm{H}$ and Peng Y: DL-3-n-butylphthalide delays the onset and progression of diabetic cataract by inhibiting oxidative stress in rat diabetic model. Sci Rep 6: 19396, 2016.

17. Lei H, Zhao CY, Liu DM, Zhang Y, Li L, Wang XL and Peng Y: 1-3-n-Butylphthalide attenuates $\beta$-amyloid-induced toxicity in neuroblastoma SH-SY5Y cells through regulating mitochondrion-mediated apoptosis and MAPK signaling. J Asian Nat Prod Res 16: 854-864, 2014.

18. Xing M, Sun Q, Wang Y, Cheng Y and Zhang N: Hydroxysafflor yellow A increases BDNF and NMDARs in the hippocampus in A vascular dementia rat model. Brain Res 1642: 419-425, 2016.

19. Deacon RM and Rawlins JN: T-maze alternation in the rodent. Nat Protoc 1: 7-12, 2006.

20. Pulsinelli WA, Brierley JB and Plum F: Temporal profile of neuronal damage in a model of transient forebrain ischemia. Ann Neurol 11: 491-498, 1982.

21. Levine B, Mizushima N and Virgin HW: Autophagy inimmunity and inflammation. Nature 469, 323-335, 2011.

22. Zhao Y, Huang G, Chen S, Gou Y, Dong Z and Zhang X: Homocysteine aggravates cortical neural cell injury through neuronal autophagy overactivation following rat cerebral ischemia-reperfusion. Int J Mol Sci 17: E1196 2016.

23. Zou W, Song Y, Li Y, Du Y, Zhang X and Fu J: The role of autophagy in the correlation between neuron damage andcognitive impairmentin ratchronic cerebral hypoperfusion. Mol Neurobiol 55: 776-791, 2018.

24. Li L, Chen J, Sun S, Zhao J, Dong X and Wang J: Effects of estradiol on autophagy and Nrf-2/ARE signals after cerebral ischemia. Cell Physiol Biochem 41: 2027-2036, 2017.

25. Kim YC and Guan KL: mTOR: A pharmacologic target for autophagy regulation. J Clin Invest 125: 25-32, 2015.

26. Wang D, Lin Q, Su S, Liu K, Wu Y and Hai J: URB597 improves cognitive impairment induced by chronic cerebral hypoperfusion by inhibiting mTOR-dependent autophagy. Neuroscience 344, 293-304, 2017.

27. Klionsky DJ, Abdalla FC, Abeliovich H, Abraham RT, Acevedo-Arozena A, Adeli K, Agholme L, Agnello M, Agostinis P, Aguirre-Ghiso JA, et al: Guidelines for the use and interpretation of assays for monitoring autophagy in higher eukaryotes. Autophagy 8: 445-544, 2012.

28. Wang J, Meng F, Cottrell JE,SacktorTC and Kass IS: Metabotropic actions of the volatile anaesthetic sevoflurane increase protein kinase $M$ synthesis and induce immediate preconditioning protection of rat hippocampal slices. J Physiol 590: 4093-4107, 2012.

29. Hölscher C: Time space and hippocampal functions. Rev Neurosci 14: 253-284, 2003.

30. Kiriyama Y and Nochi H: The function of autophagy in neurodegenerative diseases. Int J Mol Sci 16: 26797-26812, 2015.

31. Che H, Yan Y, Kang XH, Guo F, Yan ML, Liu HL, Hou X, Liu T, Zong DK, Sun LL, et al: MicroRNA-27a promotes inefficient lysosomal clearance in the hippocampi of rats following chronic brain hypoperfusion. Mol Neurobiol 54: 2595-2610, 2017.

32. Jia Y, Jin W, Xiao Y, Dong Y, Wang T, Fan M, Xu J, Meng N, Li L and Lv P: Lipoxin A4 methyl ester alleviates vascular cognition impairment by regulating the expression of proteins related to autophagy and ER stress in the rat hippocampus. Cell Mol Biol Lett 20: 475-487, 2015.

33. Yang X, He C, Liu P, Song X, Thomas T, Tshimanga S, Wang F, Niu J, Sun T and Li PA: Inhibition of mTOR pathway by rapamycin reduces brain damage in rats subjected to transient forebrain ischemia. Int J Biol Sci 11: 1424-1435, 2015. 